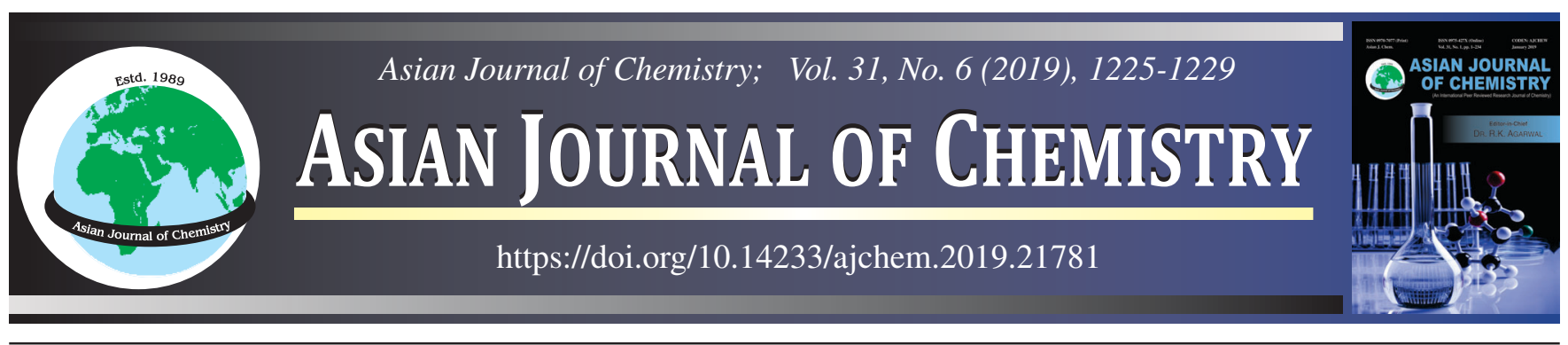

\title{
Synthesis, Anti-inflammatory, Analgesic and Antipyretic Activity of Novel 1,3,5-Trisubstituted Pyrazole Derivatives
}

Thatavarthi Padmini" and Bigala Raj Kamal

Department of Chemistry, Mewar University, Chittorgarh-312901, India

*Corresponding author: E-mail: thatap@yahoo.com

Received: 29 October 2018;

Accepted: 24 December 2018;

Published online: 29 April 2019;

AJC-19359

A novel series of 1,3,5-trisubstituted pyrazole derivatives were synthesized by the cycloaddition reaction between the electron rich $N$-substituted aryl hydrazones and nitro-olefins. Different $N$-substituted aryl hydrazones (1a-j) on reacting with 4-methyl- $\beta$-nitrostyrene (2) in ethylene glycol or trifluoroethanol-trifluoro acetic acid mixture (for acid stable hydrazones) at $120{ }^{\circ} \mathrm{C}$, yielded various $1,3,5$ trisubstituted pyrazoles (3a-j). The final products were characterized by spectral analysis using Mass, NMR and IR spectroscopy. All the products were assessed for their anti-inflammatory, analgesic and antipyretic activities on Swiss albino rats. All the compounds (3a-j) exhibited noteworthy defence against inflammation, nociception and hyperthermia. Compounds (3e and $\mathbf{3 h})$ disclosed better antiinflammatory, analgesic and antipyretic activities while compound $\mathbf{3 c}$ had highest anti-inflammatory activity compared to the standard nimesulide.

Keywords: Cycloaddition, Nitro olefins, Hydrazones, Anti-inflammatory, Analgesic, Antipyretic.

\section{INTRODUCTION}

Inflammation, pain and fever are the common problems over the sphere. In spite of the fact that numerous drugs are accessible in the market, there is dependably a chase for the new agents for the management of these problems [1]. NSAID are the essential selection of medications for inflammation, pain and are subsuming unwanted responses like gastric disturbances. Extensive research is climbing up to culminate these side effects [2].

Poly substituted pyrazole based drugs are acquiring significance in medicinal and natural products research by virtue of its multifarious properties and broad spectrum of applications for various ailments [3]. Particularly 1,3,5-trisubstituted pyrazoles build a crucial place in pharmaceutical industry as they constitute the fundamental structure for many commercial drugs such as celecoxib, sildenafil and rimonabant.

The plenteous literature affirms the ubiquitous nature of aza-heterocyclic compounds especially the pyrazole derivatives. They are documented to possess anti-inflammatory [47], antibacterial, antifungal [8-11], antiviral [12,13], antimalarial [14,15], analgesic [16-18], antipyretic [19,20], antidepressant [21-23], anticonvulsant [24,25], antidiabetic [26,27], antiangio-genetic [28], antitubercular [29] and anticancer [30-33] activities.

Since the inception of foremost synthetic method of pyrazole i.e. Paal-Knorr synthesis by condensation of 1,3-diketones with hydrazine which yield 1,3,5-trisubstituted pyrazoles, it is the subject of interest for researchers to synthesize the 1,3,5trisubstituted pyrazoles. Due to the convenience and versatility, Paal-Knorr synthesis had been the principal synthetic method for pyrazole synthesis. There are many accustomed approaches for the synthesis of various pyrazole derivatives. A bunch of literature in connection to the modification of Paal-Knorr synthesis with better yields, reported by researchers, by supplanting one of the starting material 1,3-diketone with acetylenic and olefinic ketone moieties. Distinctive reagents have been developed for synthesizing diverse pyrazole cross breeds. To swamp the harsh reaction conditions and to have regioselectivity, a basic and beneficial strategy was utilized for the synthesis of 1,3,5-trisubstituted pyrazoles [34].

In this regard, an endeavor was made to integrate a novel series of 1,3,5-trisubstituted pyrazoles by cycloaddition of $N$ arylhydrazones and nitro olefins to screen for anti-inflammatory

This is an open access journal, and articles are distributed under the terms of the Creative Commons Attribution-NonCommercial-ShareAlike 4.0 (CC BY-NC-SA 4.0) International License which allows readers to freely read, download, copy, distribute, print, search, or link to the full texts of its articles and to use them for any other lawful non-commercial purpose as long as the original source is duly acknowledged. 
(by carrageenan induced rodent paw edema model), analgesic (acetic acid induced writhing model and hot plate method) and antipyretic (yeast induced pyrexia model) in Swiss albino rats.

\section{EXPERIMENTAL}

All the solvents and reagents were procured from SigmaAldrich and purified wherever necessary. Melting points were uncorrected and determined in open capillary Electro thermal REMI CT-30 capillary melting point apparatus. NMR spectra were recorded on Bruker $300 \mathrm{MHz}$ using $\mathrm{CDCl}_{3}$. IR was recorded on Shimadzu-8400 spectrometer using $\mathrm{KBr}$ as medium. EI mass spectra were recorded on JEOL SX 102/DA-6000 mass spectrometer in positive mode.

Adult Wistar rats (180-200 g) were employed for the experiments. They were catered from Jeeva Life Sciences, Hyderabad. After stochastically dividing into different groups, the rats were adopted to the conditions for a period of 10 days before initiation of the experiment. Animals were accommodated in polypropylene cages and maintained under standard environmental conditions such as temperature $\left(26 \pm 2{ }^{\circ} \mathrm{C}\right)$ and relative humidity (45-55 \%) for $12 \mathrm{~h}$ dark/light cycle. The rats were fed with rodent pellet diet (Golden Mohur Lipton India Ltd.) and water ad libitum. The study protocol was approved by the institutional animal ethics committee (IAEC) before the commencement of experiment (1292/ac/09/CPCSEA).
General procedure for the synthesis of pyrazole derivatives

Method 1: Each, exactly weighed $1 \mathrm{mmol}$ of 4-methyl$\beta$-nitrostyrene (163 mg) and previously prepared different $N$ substituted hydrazones were dissolved in $10 \mathrm{~mL}$ of ethylene glycol. The reaction was carried at reflux conditions $\left(120^{\circ} \mathrm{C}\right)$ in an open environment for $16 \mathrm{~h}$. After cooling to room temperature, workup has been done by portioning the reaction mixture in ethyl acetate and brine solution. Ethyl acetate layer was collected, dried and crude purified over column chromatography eluting with $n$-hexane and ethyl acetate (Scheme-I).

Method 2: 4-Methyl- $\beta$-nitrostyrene ( $1 \mathrm{mmol}, 163 \mathrm{mg}$ ) and acid stable previously prepared hydrazones $(1 \mathrm{mmol})$ were dissolved in $10 \mathrm{~mL}$ of trifluoroethanol. To this mixture $0.77 \mathrm{~mL}$ of trifluoroacetic acid (TFA) was added drop wise with continuous stirring at room temperature for $48 \mathrm{~h}$. After cooling to room temperature, workup has been done by portioning the reaction mixture in ethyl acetate and brine solution. Ethyl acetate layer was collected, dried and crude product was purified over column chromatography eluting with $n$-hexane and ethyl acetate [35] (Scheme-I).

\section{Spectral data}

3-(4-Chlorophenyl)-1-(4-methoxy-phenyl)-5-p-tolyl$1 \boldsymbol{H}$-pyrazole (3a): Pale yellow solid, yield: $60 \%$, m.p. 165 ${ }^{\circ} \mathrm{C},{ }^{1} \mathrm{H}$ NMR $\left(400 \mathrm{MHz}, \mathrm{CDCl}_{3}\right), \delta=7.35(\mathrm{dt}, J=8.5,2.0 \mathrm{~Hz}$, $2 \mathrm{H}), 7.84(\mathrm{dt}, J=8.5,2.0 \mathrm{~Hz}, 2 \mathrm{H}), 6.86(\mathrm{dt}, J=9.0,2.2 \mathrm{~Hz}$,<smiles>[R]C=NN[R8]</smiles>

$\mathrm{N}$-aryl hydrazone<smiles>Cc1ccc(/C=C/[N+](=O)[O-])cc1</smiles>

4-Methyl nitrostyrene

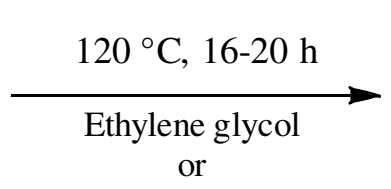

Trifluoroethanol - TFA<smiles>[R]c1cc(-c2ccc(C)cc2)n([R])n1</smiles>

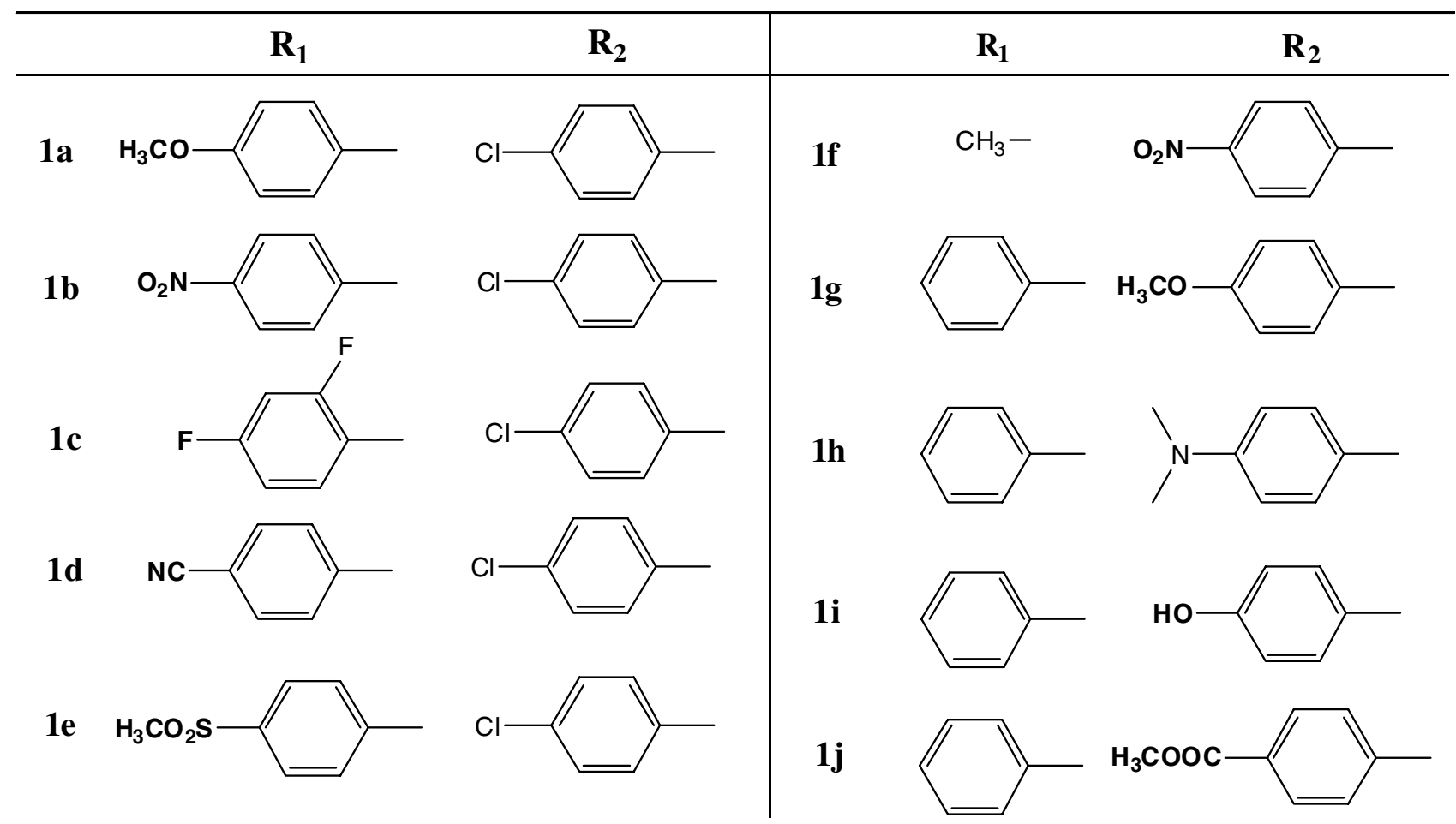

Scheme-I: Synthesis for 1,3,5-trisubstituted pyrazoles 
2H), $7.25(\mathrm{dt}, J=9.0,2.2 \mathrm{~Hz}, 2 \mathrm{H}), 7.16-7.1(\mathrm{~m}, 4 \mathrm{H}), 6.74(\mathrm{~s}$, $1 \mathrm{H}), 2.35(\mathrm{~s}, 3 \mathrm{H}), 3.82(\mathrm{~s}, 3 \mathrm{H}) ;{ }^{13} \mathrm{C}$ NMR $\left(125.7 \mathrm{MHz}, \mathrm{CDCl}_{3}\right)$ : $55.5,21.2,158.9,150.4,144.6,138.2,133.5,133.4,131.8$, $129.1,128.7,128.5,127.5,127.0,126.7,114.1,104.2$; ESIMS $(m / z):[\mathrm{M}+\mathrm{H}]^{+}$for $\mathrm{C}_{23} \mathrm{H}_{20} \mathrm{~N}_{2} \mathrm{OCl}$ is 375.1 .

3-(4-Chlorophenyl)-1-(4-nitro-phenyl)-5-p-tolyl-1Hpyrazole (3b): Yield: $15 \%$, m.p. $217^{\circ} \mathrm{C},{ }^{1} \mathrm{H}$ NMR $(400 \mathrm{MHz}$, $\left.\mathrm{CDCl}_{3}\right), \delta=8.20(\mathrm{dt}, J=9.0,2.0 \mathrm{~Hz}, 2 \mathrm{H}), 7.82(\mathrm{dt}, J=8.4$, $2.0 \mathrm{~Hz}, 2 \mathrm{H}), 7.55(\mathrm{dt}, J=9.0,2.0 \mathrm{~Hz}, 2 \mathrm{H}), 7.41(\mathrm{dt}, J=8.6$, $2.0 \mathrm{~Hz}, 2 \mathrm{H}), 6.80(\mathrm{~s}, 1 \mathrm{H}), 2.42(\mathrm{~s}, 3 \mathrm{H}), 7.22-7.18(\mathrm{~m}, 4 \mathrm{H}) .{ }^{13} \mathrm{C}$ NMR (125.7 MHz, $\left.\mathrm{CDCl}_{3}\right)$ : 21.3152.1, 145.9, 144.9, 139.4, 134.4, 131.0, 129.7, 129.0, 128.7, 127.2, 127.0, 124.5, 124.4, 106.8; HRMS-ESI $(m / z)$ : $[\mathrm{M}+\mathrm{H}]^{+}$for $\mathrm{C}_{22} \mathrm{H}_{17} \mathrm{~N}_{3} \mathrm{O}_{2} \mathrm{Cl}$ is 390.1 .

3-(4-Chlorophenyl)-1-(2,4-difluoro-phenyl)-5-p-tolyl$1 \mathrm{H}$-pyrazole (3c): Yield: $75 \%$, m.p. $167{ }^{\circ} \mathrm{C},{ }^{1} \mathrm{H}$ NMR (400 $\left.\mathrm{MHz}, \mathrm{CDCl}_{3}\right), \delta=7.36(\mathrm{dt} J=8.5,2.0 \mathrm{~Hz}, 2 \mathrm{H}), 7.80(\mathrm{dt}, J=$ 8.5, 2.0 Hz, 2H), 7.17-7.10 (m, 4H), 7.55-7.45 (m, 1H), 7.00$6.90(\mathrm{~m}, 1 \mathrm{H}), 6.90-6.84(\mathrm{~m}, 1 \mathrm{H}), 2.34(\mathrm{~s}, 3 \mathrm{H}), 6.76(\mathrm{~s}, 1 \mathrm{H})$; 21.2. ${ }^{13} \mathrm{C}$ NMR $\left(125.7 \mathrm{MHz}, \mathrm{CDCl}_{3}\right): 162.5\left(\mathrm{dd}, J_{\mathrm{C}-\mathrm{F}}=255.9\right.$, $12.6 \mathrm{~Hz}), 162.5$ (dd, $J_{\mathrm{CF}} 151.7,146.7,138.6,133.9,131.4$, $130.1\left(\mathrm{~d}, J_{\mathrm{C}-\mathrm{F}}=9.8 \mathrm{~Hz}\right), 129.3,128.8,127.7,127.1,126.8$, $124.9\left(\mathrm{dd}, J_{\mathrm{C}-\mathrm{F}}=12.3,3.9 \mathrm{~Hz}\right), 111.9\left(\mathrm{dd}, J_{\mathrm{C}-\mathrm{F}}=22.4,3.8 \mathrm{~Hz}\right)$, $\left.\left.105.1\left(\mathrm{dd}, J_{\mathrm{C}-\mathrm{F}}=26.3,23.6 \mathrm{~Hz}\right), 103.9,251.6,11.1 \mathrm{~Hz}\right), \mathrm{Hz}\right)$, $111.9\left(\mathrm{dd}, J_{\mathrm{C}-\mathrm{F}}=22.4,3.8 \mathrm{~Hz}\right), 105.1\left(\mathrm{dd}, J_{\mathrm{C}-\mathrm{F}}=26.3,23.6\right.$ $\mathrm{Hz}), 103.9,21.2$; ESI-MS $(\mathrm{m} / \mathrm{z})$ : $[\mathrm{M}+\mathrm{H}]^{+}$for $\mathrm{C}_{22} \mathrm{H}_{16} \mathrm{~N}_{2} \mathrm{~F}_{2} \mathrm{Cl}$, 381.

4-[3-(4-Chlorophenyl)-5-p-tolyl-pyrazol-1-yl]benzonitrile (3d): Yield: $50 \%$, m.p. $186{ }^{\circ} \mathrm{C},{ }^{1} \mathrm{H}$ NMR $(400$ $\left.\mathrm{MHz}, \mathrm{CDCl}_{3}\right), \delta=7.80(\mathrm{dt}, J=8.5,2.0 \mathrm{~Hz}, 2 \mathrm{H}), 7.40(\mathrm{dt}, J=$ $8.5,2.0 \mathrm{~Hz}, 2 \mathrm{H}), 7.60(\mathrm{dt}, J=8.7,2.0 \mathrm{~Hz}, 2 \mathrm{H}), 7.46(\mathrm{dt}, J=$ $8.7,2.0 \mathrm{~Hz}, 2 \mathrm{H}), 2.40(\mathrm{~s}, 3 \mathrm{H}), 7.22-7.13(\mathrm{~m}, 4 \mathrm{H}), 6.76(\mathrm{~s}$, $1 \mathrm{H}) ;{ }^{13} \mathrm{C} \mathrm{NMR}\left(125.7 \mathrm{MHz}, \mathrm{CDCl}_{3}\right): 21.3,151.9,145.1,143.4$, 139.2, 134.2, 132.8, 131.0,129.6, 128.9, 128.7, 127.1, 127.0, 124.8, 118.3, 110.5, 106.5; ESI-MS $(\mathrm{m} / \mathrm{z}):[\mathrm{M}+\mathrm{H}]^{+}$for $\mathrm{C}_{14} \mathrm{H}_{16} \mathrm{~N}_{2} \mathrm{Cl}$ is 247.1 .

3-(4-Chlorophenyl)-1-(4-methanesulfonyl-phenyl)-5-ptolyl-1H-pyrazole (3e): Yield: $60 \%$, m.p. $210{ }^{\circ} \mathrm{C},{ }^{1} \mathrm{H}$ NMR $\left(400 \mathrm{MHz}, \mathrm{CDCl}_{3}\right), \delta=7.55(\mathrm{dt}, J=8.5,1.8 \mathrm{~Hz}, 2 \mathrm{H}), 7.90$ (dt, $J=8.5,1.8 \mathrm{~Hz}, 2 \mathrm{H}), 7.42(\mathrm{dt}, J=8.5,1.8 \mathrm{~Hz}, 2 \mathrm{H}), 7.85$ $(\mathrm{dt}, J=8.5,1.8 \mathrm{~Hz}, 2 \mathrm{H}), 6.77(\mathrm{~s}, 1 \mathrm{H}), 3.04(\mathrm{~s}, 3 \mathrm{H}), 2.40(\mathrm{~s}$, 3H), 7.20-7.15 (m, 4H); ${ }^{13} \mathrm{C} \mathrm{NMR}\left(125.7 \mathrm{MHz}, \mathrm{CDCl}_{3}\right)$ : 44.5, 21.3.151.9, 145.1, 144.2, 139.2, 138.5, 134.2, 131.0, 129.6, 128.9, 128.6, 128.3, 127.1, 126.9, 124.9, 106.4; ESI-MS ( $\mathrm{m} / \mathrm{z})$ : $[\mathrm{M}+\mathrm{H}]^{+}$for $\mathrm{C}_{23} \mathrm{H}_{20} \mathrm{~N}_{2} \mathrm{O}_{2} \mathrm{SCl}, 423.1$

1-Methyl-3-(4-nitrophenyl)-5-p-tolyl-1 $H$-pyrazole (3f): Yield: $52 \%$, m.p. $158{ }^{\circ} \mathrm{C},{ }^{1} \mathrm{H}$ NMR $\left(400 \mathrm{MHz}, \mathrm{CDCl}_{3}\right), \delta=$ $7.67(\mathrm{dt}, J=9.0,2.0 \mathrm{~Hz}, 2 \mathrm{H}), 8.10(\mathrm{dt}, J=9.0,2.0 \mathrm{~Hz}, 2 \mathrm{H})$, 7.16-7.10 (m, 4H), $7.44(\mathrm{~s}, 1 \mathrm{H}) ;{ }^{13} \mathrm{C}$ NMR $(125.7 \mathrm{MHz}$, $\mathrm{CDCl}_{3}$ ): 39.2, 21.1.146.8, 146.1, 140.3, 137.0, 130.8, 129.5, 129.4, 128.6, 128.4, 123.5, 122.0, MS $(\mathrm{m} / \mathrm{z}):[\mathrm{M}+\mathrm{H}]^{+}$for $\mathrm{C}_{17} \mathrm{H}_{16} \mathrm{~N}_{3} \mathrm{O}_{2}$ is 294.1 .

3-(4-Methoxyphenyl)-1-phenyl-5-p-tolyl-1H-pyrazole (3g): Yield: $75 \%$, m.p. $162{ }^{\circ} \mathrm{C},{ }^{1} \mathrm{H}$ NMR $\left(400 \mathrm{MHz}, \mathrm{CDCl}_{3}\right)$, $\delta=7.42-7.26(\mathrm{~m}, 4 \mathrm{H}), 7.84(\mathrm{dt}, J=8.8,2.0 \mathrm{~Hz}, 2 \mathrm{H}), 7.16(\mathrm{dt}$, $J=8.2,1.8 \mathrm{~Hz}, 2 \mathrm{H}), 7.11(\mathrm{~d}, J=8.0 \mathrm{~Hz}, 2 \mathrm{H}), 6.96(\mathrm{dt}, J=$ $8.8,2.0 \mathrm{~Hz}, 2 \mathrm{H}), 6.72(\mathrm{~s}, 1 \mathrm{H}), 3.85(\mathrm{~s}, 3 \mathrm{H}), 2.35(\mathrm{~s}, 3 \mathrm{H}),{ }^{13} \mathrm{C}$ NMR (125.7 MHz, $\left.\mathrm{CDCl}_{3}\right)$ : 55.2, 21.2159.6, 151.7, 144.4, 140.3, 138.2, 129.1, 128.8, 128.6, 127.8, 127.2, 127.1, 126.0,
125.3, 114.0, 104.6, ESI-MS $(m / z):[\mathrm{M}+\mathrm{H}]^{+}$for $\mathrm{C}_{23} \mathrm{H}_{21} \mathrm{~N}_{2} \mathrm{O}$ is 341.2 .

Dimethyl-[4-(1-phenyl-5-p-tolyl-1H-pyrazol-3-yl)phenyl]amine (3h): Yield: $55 \%$, m.p. $184{ }^{\circ} \mathrm{C},{ }^{1} \mathrm{H}$ NMR (400 $\left.\mathrm{MHz}, \mathrm{CDCl}_{3}\right), \delta=7.80(\mathrm{dt}, J=9.0,2.0 \mathrm{~Hz}, 2 \mathrm{H}), 7.15(\mathrm{dt}, J=$ $8.2,1.7 \mathrm{~Hz}, 2 \mathrm{H}), 7.09$ (d, $J=8.2 \mathrm{~Hz}, 2 \mathrm{H}), 6.77$ (dt, $J=9.0$, $2.0 \mathrm{~Hz}, 2 \mathrm{H}), 6.70(\mathrm{~s}, 1 \mathrm{H}), 2.32(\mathrm{~s}, 3 \mathrm{H}), 7.40-7.20(\mathrm{~m}, 5 \mathrm{H})$, $2.96(\mathrm{~s}, 6 \mathrm{H}) ;{ }^{13} \mathrm{C}$ NMR $\left(125.7 \mathrm{MHz}, \mathrm{CDCl}_{3}\right): 40.5,21.2,152.3$, 150.4, 144.1, 140.4, 138.0, 129.1, 128.8, 128.6, 128.0, 127.0, 126.7, 125.3, 121.5, 112.4, 104.3; ESI-MS ( $/ \mathrm{m} / \mathrm{z}):[\mathrm{M}+\mathrm{H}]^{+}$for. $\mathrm{C}_{24} \mathrm{H}_{24} \mathrm{~N}_{3}$ is 354.2 .

3-(1-Phenyl-5-p-tolyl-1H-pyrazol-3-yl)phenol (3i): Yield: $80 \%$, m.p. $212{ }^{\circ} \mathrm{C},{ }^{1} \mathrm{H}$ NMR $\left(400 \mathrm{MHz}, \mathrm{CDCl}_{3}\right), \delta=$ 7.40-7.34 (m, 2H), 7.05 (br. s, $1 \mathrm{H}), 6.74$ (dd, $J=8.0,2.3 \mathrm{~Hz}$, $1 \mathrm{H}), 6.70(\mathrm{~s}, 1 \mathrm{H}), 2.32(\mathrm{~s}, 3 \mathrm{H}), 7.12-7.06(\mathrm{~m}, 4 \mathrm{H}), 7.34-7.17$ $(\mathrm{m}, 6 \mathrm{H}) ;{ }^{13} \mathrm{C}$ NMR $\left(125.7 \mathrm{MHz}, \mathrm{CDCl}_{3}\right): 21.2 .156 .3,151.8$, 144.6, 139.9, 138.3, 134.1, 129.8, 129.1, 128.9, 128.5, 127.5, 127.4, 125.4, 118.1, 115.4, 112.9,105.1, ESI-MS $(\mathrm{m} / \mathrm{z})$ : $[\mathrm{M}+\mathrm{H}]^{+}$for $\mathrm{C}_{22} \mathrm{H}_{19} \mathrm{~N}_{2} \mathrm{O}$ is 327.1 .

4-(1-Phenyl-5-p-tolyl-1H-pyrazol-3-yl)benzoic acid methyl ester (3j): Yield: $30 \%$, m.p. $152{ }^{\circ} \mathrm{C},{ }^{1} \mathrm{H}$ NMR (400 $\mathrm{MHz}, \mathrm{CDCl}_{3}$ ), $\delta=7.99(\mathrm{dt}, J=8.4,1.8 \mathrm{~Hz}, 2 \mathrm{H}), 8.10(\mathrm{dt}, J=$ $8.4,1.8 \mathrm{~Hz}, 2 \mathrm{H}), 6.84(\mathrm{~s}, 1 \mathrm{H}), 3.93(\mathrm{~s}, 3 \mathrm{H}), 2.35$ (s, 3H), 7.26$7.10(\mathrm{~m}, 4 \mathrm{H}), 7.48-7.28(\mathrm{~m}, 5 \mathrm{H}) ;{ }^{13} \mathrm{C}$ NMR $(125.7 \mathrm{MHz}$, $\left.\mathrm{CDCl}_{3}\right): 52.0,21.2,166.9,150.7,144.7,140.0,138.4,137.5$, $129.9,129.2,129.2,128.9,128.5,127.5,127.3,125.5,125.2$, 105.3, ESI-MS $(m / z):[\mathrm{M}+\mathrm{H}]^{+}$for $\mathrm{C}_{24} \mathrm{H}_{21} \mathrm{~N}_{2} \mathrm{O}_{2}$ is 369 .

Anti-inflammatory activity (carrageenan-induced rat paw oedema test): All the synthesized pyrazoles (3a-j) were administered in two doses of $50 \mathrm{mg} / \mathrm{kg}$ body weight based upon their acute toxicity studies using standard protocols [36]. Nimesulide at a dose of $50 \mathrm{mg} / \mathrm{kg}$ body weight was served as reference standard. The compounds were suspended in $0.5 \%$ carboxymethyl cellulose (CMC) and administered orally to the Wister rats. The control animals received $0.5 \%$ carboxymethyl cellulose. After $30 \mathrm{~min}$ drug administration, $0.1 \mathrm{~mL}$ of $1 \%$ carrageenan in normal saline solution was injected into the sub plantar region of one of the hind paws. The paw oedema volume was recorded using a plethysmometer (UGO Basile, Italy) at different time intervals.

Analgesic activity (acetic acid-induced writhing model): This experiment was carried out by standard procedures using Wistar rats of either sex [37]. Three groups of six animals each served as control, test and standard. The control group was administered with the normal saline solution, the test group supplied with $50 \mathrm{mg} / \mathrm{kg}$ body weight of the compounds. The standard group was administered with nimesulide at a dose of $50 \mathrm{mg} / \mathrm{kg}$ prepared in water. Food was restricted during experiments. Writhings were induced $30 \mathrm{~min}$ after the last dose by intraperitoneal injection of $10 \mathrm{~mL} / \mathrm{kg}$ of $0.6 \%$ acetic acid in distilled water. The number of writhings that is a number of abdominal contractions, trunk twist responses and extension of hind limbs was recorded immediately for a period of 10 min.

Antipyretic activity (yeast-induced pyrexia model): The antipyretic activity of the test compounds was screened by yeast-induced pyrexia model in rats [38]. The rectal temperature of each rat was recorded at predetermined time intervals. 
Fever was induced by injecting $15 \%$ Brewer's yeast (Saccharomyces cerevisiae) suspension. The animals were acclimatized for some time. A thermistor probe was inserted 3-4 cm deep into the rectum after fastening the tail to record the basal rectal temperature. The animals were then given a subcutaneous injection of $10 \mathrm{~mL} / \mathrm{kg}$ of $15 \% \mathrm{w} / \mathrm{v}$ Brewer's yeast suspended in $0.5 \% \mathrm{w} / \mathrm{v}$ carboxymethyl cellulose solution and the animals were returned to their housing cages. After $19 \mathrm{~h}$, yeast injection, the rats were again restrained in individual cages to record their rectal temperature before and at $1 \mathrm{~h}$ intervals up to $3 \mathrm{~h}$ after the administration of prescribed dose of test compounds, vehicle and paracetamol $(50 \mathrm{mg} / \mathrm{kg})$.

\section{RESULTS AND DISCUSSION}

All the synthesized 1,3,5-trisubstituted pyrazoles were evaluated for anti-inflammatory, analgesic and antipyretic activities in rats.

Anti-inflammatory activity: The experimental results were compared with the standard drug nimesulide $(50 \mathrm{mg} / \mathrm{kg})$ states that the 1,3,5-trisubstituted pyrazoles had shown substantial antiinflammatory activity in carrageenan-induced rat paw oedema test at a dose of $50 \mathrm{mg} / \mathrm{kg}$ (Table-1). Compound $\mathbf{3 c}$ had the highest anti-inflammatory activity (41\% after $3 \mathrm{~h}$ ) and compound $\mathbf{3 h}$ had rapid onset of action (17.5\% in $1 \mathrm{~h}$ ) than the other pyrazoles. However, the maximum inhibition of oedema was produced by compounds $\mathbf{3 c}, \mathbf{3 b}$ and $\mathbf{3 d}$ as compared to standard nimesulide (44.8\% after $3 \mathrm{~h}$ ).

Analgesic activity: Similarly, all the prepared pyrazole derivatives had shown significant inhibition of acetic acid induced writhings in rats at a dose of $50 \mathrm{mg} / \mathrm{kg}$ (Table-1) when compared with the standard drug nimesulide $(50 \mathrm{mg} / \mathrm{kg})$. However, the maximum inhibition of writhings was produced by compounds $3 \mathbf{h}$ ( $52.9 \%$ of inhibition), 3e (52.6\% of inhibition) and $\mathbf{3 i}$ (48.7\% of inhibition) as compared to standard nimesulide (57.9\% of inhibition).

Antipyretic activity: Similar to the anti-inflammatory activity results, all the derivatives of pyrazoles had shown significant antipyretic activity in yeast induced rat pyrexia test at a dose of $50 \mathrm{mg} / \mathrm{kg}$ (Table-2) when compared with the standard drug nimesulide $(50 \mathrm{mg} / \mathrm{kg})$. Among all the synthesized pyrazoles, Compound $\mathbf{3 h}$ had the highest antipyretic activity (36.03 \pm 0.14 after $3 \mathrm{~h})$ and rapid onset of action $(37.39 \pm 0.27$ in $1 \mathrm{~h})$ than the other synthesized compounds. However, the maximum inhibition of temperature was produced by compounds $\mathbf{3 h}, \mathbf{3 i}$, 3c and $\mathbf{3 e}$ as compared to standard nimesulide $(35.45 \pm 0.21$ after $3 \mathrm{~h})$.

TABLE-1

MICROBIAL ACTIVITY OF 1,3,5-TRISUBSTITUTED PYRAZOLES IN CARRAGEENAN-INDUCED RAT PAW OEDEMA TEST

\begin{tabular}{|c|c|c|c|c|c|c|c|}
\hline \multirow{3}{*}{ Compd. } & \multirow{3}{*}{ Dose $(\mathrm{mg} / \mathrm{kg})$} & \multicolumn{4}{|c|}{ Anti-inflammatory activity } & \multicolumn{2}{|c|}{ Analgesic activity } \\
\hline & & \multicolumn{2}{|c|}{$\begin{array}{l}\text { Mean paw volume at different } \\
\text { time intervals }(\mathrm{mL})\end{array}$} & \multicolumn{2}{|c|}{$\begin{array}{l}\text { Percentage inhibition of } \\
\text { edema volume }\end{array}$} & \multirow{2}{*}{$\begin{array}{l}\text { Writhing } \\
\text { response }\end{array}$} & \multirow{2}{*}{$\begin{array}{l}\text { Percentage } \\
\text { inhibition }\end{array}$} \\
\hline & & $1 \mathrm{~h}$ & $3 \mathrm{~h}$ & $1 \mathrm{~h}$ & $3 \mathrm{~h}$ & & \\
\hline Control & 50 & $5.87 \pm 0.052$ & $6.85 \pm 0.012$ & 0.0 & 0.0 & $37.12 \pm 0.54$ & 0.0 \\
\hline Nimesulide & 50 & $4.82 \pm 0.027$ & $3.78 \pm 0.024$ & 17.9 & 44.8 & $15.63 \pm 0.71$ & 57.9 \\
\hline $3 a$ & 50 & $5.12 \pm 0.047^{* *}$ & $5.26 \pm 0.044$ & 12.8 & 23.2 & $24.31 \pm 0.65^{* *}$ & 34.5 \\
\hline $\mathbf{3 b}$ & 50 & $5.02 \pm 0.064^{* *}$ & $4.12 \pm 0.056^{* *}$ & 14.5 & 39.9 & $21.04 \pm 1.47$ & 44.5 \\
\hline $3 c$ & 50 & $4.90 \pm 0.051^{* *}$ & $4.04 \pm 0.061^{* *}$ & 16.5 & 41.0 & $17.25 \pm 0.83^{* *}$ & 43.3 \\
\hline 3d & 50 & $5.04 \pm 0.018$ & $4.19 \pm 0.043^{* *}$ & 14.1 & 38.8 & $21.44 \pm 0.32^{* *}$ & 42.2 \\
\hline $3 e$ & 50 & $4.98 \pm 0.048^{* *}$ & $3.97 \pm 0.067$ & 15.2 & 20.3 & $17.59 \pm 0.97^{* *}$ & 52.6 \\
\hline $3 f$ & 50 & $5.06 \pm 0.013^{* *}$ & $4.32 \pm 0.049^{* *}$ & 13.8 & 36.9 & $24.36 \pm 1.55$ & 34.4 \\
\hline $3 g$ & 50 & $5.09 \pm 0.045$ & $4.57 \pm 0.023^{* *}$ & 13.3 & 33.3 & $19.73 \pm 1.65^{* *}$ & 46.8 \\
\hline $3 \mathrm{~h}$ & 50 & $4.84 \pm 0.041^{* * *}$ & $4.46 \pm 0.018^{* * *}$ & 17.5 & 34.8 & $17.50 \pm 1.38^{* *}$ & 52.9 \\
\hline $3 \mathbf{i}$ & 50 & $5.13 \pm 0.090^{* *}$ & $4.85 \pm 0.031^{* *}$ & 12.6 & 29.2 & $19.03 \pm 1.91$ & 48.7 \\
\hline $3 \mathbf{j}$ & 50 & $5.10 \pm 0.032^{* *}$ & $4.61 \pm 0.092$ & 10.6 & 32.7 & $23.50 \pm 1.56^{* *}$ & 36.7 \\
\hline
\end{tabular}

$\mathrm{N}=6$, Values are Mean $\pm \mathrm{SEM} ;{ }^{* *} P<0.01$ (significant), values are compared with control group.

TABLE-2

ANTIPYRETIC ACTIVITY OF SYNTHESIZED 1,3,5-TRISUBSTITUTED PYRAZOLES IN YEAST INDUCED RAT PYREXIA TEST

\begin{tabular}{cccccc}
\hline \multirow{2}{*}{ Compd. } & \multirow{2}{*}{ Dose $(\mathrm{mg} / \mathrm{kg})$} & \multicolumn{4}{c}{ Yeast induced pyrexia (temperature in $\left.{ }^{\circ} \mathrm{C}\right)$} \\
\cline { 3 - 6 } & & $0 \mathrm{~h}$ & $1 / 2 \mathrm{~h}$ & $1 \mathrm{~h}$ & $3 \mathrm{~h}$ \\
\hline Control & 50 & $37.65 \pm 0.24$ & $37.48 \pm 0.22$ & $37.24 \pm 0.25$ & $36.56 \pm 0.15$ \\
Nimesulide & 50 & $37.47 \pm 0.19$ & $36.88 \pm 0.06$ & $36.67 \pm 0.02$ & $35.45 \pm 0.21$ \\
3a & 50 & $37.54 \pm 0.30$ & $37.38 \pm 1.01$ & $37.25 \pm 0.10^{* *}$ & $36.52 \pm 0.06^{*}$ \\
3b & 50 & $37.58 \pm 0.38^{* *}$ & $37.47 \pm 0.57^{* *}$ & $36.21 \pm 0.12^{* *}$ & $36.14 \pm 0.07^{* *}$ \\
3c & 50 & $37.37 \pm 0.34$ & $36.85 \pm 0.68^{* *}$ & $36.64 \pm 0.22$ & $36.10 \pm 0.18^{* *}$ \\
3d & 50 & $37.58 \pm 1.37^{* *}$ & $37.38 \pm 0.15^{*}$ & $37.16 \pm 0.17^{* *}$ & $36.69 \pm 0.06$ \\
3e & 50 & $37.46 \pm 0.22^{*}$ & $36.87 \pm 0.21^{* *}$ & $36.58 \pm 0.25$ & $36.24 \pm 0.16^{* *}$ \\
3f & 50 & $37.44 \pm 0.35$ & $37.14 \pm 0.46$ & $36.81 \pm 0.15^{* *}$ & $36.68 \pm 0.12^{* *}$ \\
3g & 50 & $37.72 \pm 0.27^{* *}$ & $37.45 \pm 0.36^{* *}$ & $37.22 \pm 0.13^{*}$ & $36.98 \pm 0.05^{*}$ \\
3h & 50 & $37.88 \pm 0.35^{*}$ & $37.39 \pm 0.27^{* *}$ & $36.32 \pm 0.15$ & $36.03 \pm 0.14^{* *}$ \\
3i & 50 & $37.74 \pm 0.27^{* *}$ & $37.08 \pm 0.12^{* *}$ & $36.55 \pm 0.18^{* *}$ & $36.12 \pm 0.18^{* *}$ \\
3j & 50 & $37.54 \pm 0.11$ & $37.34 \pm 0.16^{* *}$ & $37.15 \pm 0.07^{* *}$ & $36.61 \pm 0.02^{*}$ \\
\hline
\end{tabular}

$\mathrm{N}=6$, Values are Mean $\pm \mathrm{SEM} ;{ }^{* *} P<0.01$ (significant); ${ }^{*} P<0.05$ (significant) values are compared with control group. 


\section{Conclusion}

In conclusion, the synthesis of various 1,3,5-trisubstituted pyrazoles (3a-j) have been achieved by the cycloaddition of nitro olefins with $N$-mono substituted hydrazones with good yields. All the synthesized pyrazoles (3a-j) have been evaluated for their anti-inflammatory, analgesic and antipyretic activities. Results revealed that all the compounds had shown significant anti-inflammatory, analgesic and antipyretic activities. Compound $\mathbf{3 h}$ had shown comparatively better anti-inflammatory, analgesic and antipyretic activities and compound $\mathbf{3 c}$ had highest anti-inflammatory property compared to the standard nimesulide. There is a need to continue the research further in structural and molecular aspects to figure out the exact mechanism to develop them as potential leads for inflammation, pain and fever.

\section{CONFLICT OF INTEREST}

The authors declare that there is no conflict of interests regarding the publication of this article.

\section{REFERENCES}

1. M.A. Khan, H. Khan, S. Khan, T. Mahmood, P.M. Khan and A. Jabar, J. Enzyme Inhib. Med. Chem., 24, 632 (2009); https://doi.org/10.1080/14756360802321120.

2. S. Domiati, A. El-Mallah, A. Ghoneim, A. Bekhit and H.A. El Razik, Inflamm. Pharmacol., 24, 163 (2016); https://doi.org/10.1007/s10787-016-0270-7.

3. A. Ansari, A. Ali, M. Asif and S. Shamsuzzaman, New J. Chem., 41, 16 (2017); https://doi.org/10.1039/C6NJ03181A.

4. S.M. El-Moghazy, F.F. Barsoum, H.M. Abdel-Rahman and A.A. Marzouk, Med. Chem. Res., 21, 1722 (2012); https://doi.org/10.1007/s00044-011-9691-4.

5. R.S. Kumar, I.A. Arif, A. Ahamed and A. Idhayadhulla, Saudi J. Biol. Sci., 23, 614 (2016); https://doi.org/10.1016/j.sjbs.2015.07.005.

6. A.K. Tewari, P. Srivastava, V.P. Singh, A. Singh, R.K. Goel and C. Mohan, Chem. Pharm. Bull., 58, 634 (2010); https://doi.org/10.1248/cpb.58.634.

7. P. Mahaveer, R.G. Kulkarni, P. Radakrishna, V.M. Chandrashekar and G. Achaiah, Indian J. Chem., 52B, 818 (2013).

8. M. Rahimizadeh, M. Pordel, M. Bakavoli, S. Rezaeian and A. Sadeghian, World J. Microbiol. Biotechnol., 26, 317 (2010); https://doi.org/10.1007/s11274-009-0178-0.

9. K.P. Sharma and H.K. Sharma, Asian J. Chem., 19, 4129 (2007).

10. V.H. Bhaskar, V.S. More and M. Kumar, Asian J. Chem., 20, 5474 (2008).

11. P.R. Solanki, K.N. Wadodkar, M.K. Rai and P. Wagh, Asian J. Chem., 15, 1864 (2003).

12. J.S. Larsen, M.A. Zahran, E.B. Pedersen and C. Nielsen, Monatsh. Chem., 130, 1167 (1999); https://doi.org/10.1007/PL00010295.

13. O.I. El-Sabbagh, M.M. Baraka, S.M. Ibrahim, C. Pannecouque, G. Andrei, R. Snoeck, J. Balzarini and A.A. Rashad, Eur. J. Med. Chem., 44, 3746 (2009); https://doi.org/10.1016/j.ejmech.2009.03.038.

14. A.A. Bekhit, A. Hymete, H. Asfaw and A.E. Bekhit, Arch. Pharm. Med. Chem., 345, 147 (2012); https://doi.org/10.1002/ardp.201100078.

15. A.B. Vaidya, J.M. Morrisey, Z. Zhang, S. Das, T.M. Daly, T.D. Otto, N.J. Spillman, M. Wyvratt, P. Siegl, J. Marfurt, G. Wirjanata, B.F. Sebayang, R.N. Price, A. Chatterjee, A. Nagle, M. Stasiak, S.A.
Charman, I. Angulo-Barturen, S. Ferrer, M. Belén Jiménez-Díaz, M.S. Martínez, F.J. Gamo, V.M. Avery, A. Ruecker, M. Delves, K. Kirk, M. Berriman, S. Kortagere, J. Burrows, E. Fan and L.W. Bergman, Nat. Commun., 5, 5521 (2014); https://doi.org/10.1038/ncomms6521.

16. N.M. Abd-El Gawad, G.S. Hassan and H.H. Georgey, Med. Chem. Res., 21, 983 (2012); https://doi.org/10.1007/s00044-011-9606-4.

17. T.P. Selvam, P.V. Kumar, G. Saravanan and C.R. Prakash, J. Saudi Chem. Soc., 18, 1015 (2014); https://doi.org/10.1016/j.jscs.2011.12.006.

18. N.D. Jayanna, H.M. Vagdevi, J.C. Dharshan, R. Raghavendra and S.B. Telkar, Med. Chem. Res., 22, 5814 (2013); https://doi.org/10.1007/s00044-013-0565-9.

19. D.C. Malvar, R.T. Ferreira, R.A. de Castro, L.L. de Castro, A.C.C. Freitas, E.A. Costa, I.F. Florentino, J.C.M. Mafra, G.E.P. de Souza and F.A. Vanderlinde, Life Sci., 95, 81 (2014); https://doi.org/10.1016/j.lfs.2013.12.005.

20. G. Menozzi, L. Mosti, P. Schenone, M. D'Amico, A. Filippelli and F. Rossi, Farmaco Soc. Chimica Italiana, 47, 1495 (1992).

21. R.M. Geha, K. Chen, J. Wouters, F. Ooms and J. Shih, J. Biol. Chem., 277, 17209 (2002); https://doi.org/10.1074/jbc.M110920200.

22. P. Foley, M. Gerlach, M.B.H. Youdim and P. Riederer, Parkinsonism Relat. Disord., 6, 25 (2000); https://doi.org/10.1016/S1353-8020(99)00043-7.

23. E. Palaska, F. Aydin, G. Ucar and D. Erol, Arch. Pharm. Chem. Life Sci., 341, 209 (2008); https://doi.org/10.1002/ardp.200700159.

24. P. Aragade, K. Sadhana, K. Hemant, B. Dwarkadas and M. Veeresh, Int. J. Drug Des. Disc., 3, 688 (2012).

25. V. Alagarsamy and G. Saravanan, Med. Chem. Res., 22, 1711 (2013); https://doi.org/10.1007/s00044-012-0169-9.

26. S. Sen, B. De and T.S. Easwari, Asian J. Chem., 26, 6616 (2014); https://doi.org/10.14233/ajchem.2014.17003.

27. P.A. Datar and S.R. Jadhav, Int. J. Med. Chem., 2015, 1 (2015); https://doi.org/10.1155/2015/670181.

28. K.M. Kasiotis, E.N. Tzanetou and S.A. Haroutounian, Front Chem., 2, 1 (2014); https://doi.org/10.3389/fchem.2014.00078.

29. F.N. Kang, S. Kannan, K. Wichapong, L.C. Owono, W. Sippl and E. Megnassan, Mol. bioSyst., 10, 223 (2014); https://doi.org/10.1039/C3MB70449A.

30. I.F. Nassar, A.F. El Farargy, F.M. Abdelrazek and N.S.M. Ismail, Nucleic Acids, 36, 275 (2017); https://doi.org/10.1080/15257770.2016.1276290.

31. A. Balbi, M. Anzaldi, C. Macciò, C. Aiello, M. Mazzei, R. Gangemi, P. Castagnola, M. Miele, C. Rosano and M. Viale, Eur. J. Med. Chem., 46, 5293 (2011); https://doi.org/10.1016/j.ejmech.2011.08.014.

32. T. Ren, J. Wang, G. Li and Y. Li, Asian J. Chem., 26, 8309 (2014); https://doi.org/10.14233/ajchem.2014.16893.

33. P. Ramesh and K. Bhaskar, Asian J. Chem., 28, 2517 (2016); https://doi.org/10.14233/ajchem.2016.20061.

34. L. Knorr, Ber. Dtsch. Chem. Ges., 16, 2597 (1883); https://doi.org/10.1002/cber.188301602194.

35. X. Deng and N.S. Mani, J. Org. Chem., 73, 2412 (2008); https://doi.org/10.1021/jo7026195.

36. C.J. Morris, eds.: P.G. Winyard, C.J. Morris and D.A. Willoughby, Carrageenan-Induced Edema in the Rat and Mouse, In: Methods in Molecular Biology: Inflammation Protocols, Humana Press: New Jersey, vol. 225, 115 (2003).

37. R. Koster, M. Anderson and E.J. D. Beer, Acetic Acid for Analgesic Screening, Federation Proceedings, vol. 18, pp. 412-417 (1959).

38. S.S. Adams, P. Hebborn and J.S. Nicholas, J. Pharm. Pharmacol., 20, 305 (1968); https://doi.org/10.1111/j.2042-7158.1968.tb09744.x. 